Pacific

Journal of

Mathematics

UNIQUENESS RESULT ON NONNEGATIVE SOLUTIONS OF A LARGE CLASS OF DIFFERENTIAL INEQUALITIES ON RIEMANNIAN MANIFOLDS

YUHUA SUN 


\title{
UNIQUENESS RESULT ON NONNEGATIVE SOLUTIONS OF A LARGE CLASS OF DIFFERENTIAL INEQUALITIES ON RIEMANNIAN MANIFOLDS
}

\author{
YUHUA SUN
}

\begin{abstract}
We consider a large class of differential inequalities on complete connected Riemannian manifolds and provide a sufficient condition in terms of volume growth for the uniqueness of nonnegative solutions to the differential inequalities.
\end{abstract}

\section{Introduction}

The purpose of this paper is to give a sufficient condition for the uniqueness of nonnegative solutions to a large class of the differential inequalities

$$
L u+V(x) u^{\sigma} \leq 0
$$

on a connected geodesically complete noncompact $\mathrm{N}$-dimensional Riemannian manifold $M^{N}$ with $N \geq 2$. Here the operator $L$ is defined by

$$
L u=\operatorname{div}(A(x, u, \nabla u)),
$$

where $A(x, \eta, \xi)=\left(A_{i}(x, \eta, \xi)\right)$ is a vector field on $M^{N}$, and for $i=1, \ldots, N$ the $A_{i}(x, \eta, \xi)$ are Carathéodorian functions defined on $M^{N} \times[0, \infty) \times T M^{N}$, and $T M^{N}$ is the tangent bundle of $M^{N}$. The function $V$ is positive, measurable, and locally integrable on $M^{N}$.

Let $m \geq 1$ be an arbitrary given number. We say that the operator $L$ belongs to the class $A(m)$ if there exists a positive constant $C$ such that, for almost all $x \in M^{N}$, all $\eta \in[0, \infty)$, and all $\xi, \zeta \in T_{x} M^{N}$, the following conditions hold:

$$
\left\{\begin{array}{l}
(A(x, \eta, \xi), \xi) \geq 0 \\
|(A(x, \eta, \xi), \zeta)| \leq C(A(x, \eta, \xi), \xi)^{\frac{m-1}{m}}|\zeta|
\end{array}\right.
$$

where $(\cdot, \cdot)$ is the inner product given by the Riemannian metric, and $|\zeta|$ is the norm of $\zeta$ in $T_{x} M^{N}$.

MSC2010: primary 35J99; secondary 58J05.

Keywords: differential inequalities, Riemannian manifolds, volume growth, uniqueness. 
The definition of such a class operator $A(m)$ was first introduced by Mīklyukov [1979; 1980]. Actually, the operators of such a class are quite common. Let us mention some examples:

(1) $m$-Laplacian operator:

$$
L_{1} u=\operatorname{div}\left(|\nabla u|^{m-2} \nabla u\right), \quad m>1 .
$$

(2) Mean curvature type operators:

$$
L_{2} u=\operatorname{div}\left(\frac{|\nabla u|^{m-2} \nabla u}{\sqrt{1+|\nabla u|^{m}}}\right), \quad m>1 .
$$

and

$$
L_{3} u=\operatorname{div}\left(\frac{|\nabla u|^{m-2} \nabla u}{\sqrt{1+|\nabla u|^{2}}}\right), \quad m>1 .
$$

(3) Nonlinear operator:

$$
L_{4} u=\operatorname{div}\left(a(x, u, \nabla u)|\nabla u|^{m-2} \nabla u\right), \quad m>1 .
$$

The definition of $L$ in (1-3) is less restrictive than the one defined by

$$
|A(x, \eta, \xi)| \leq C_{1}|\xi|^{m-1}, \quad|(A(x, \eta, \xi), \xi)| \geq C_{2}|\xi|^{m},
$$

for some positive constants $C_{1}, C_{2}$. For example, by choosing $a(x, \eta, \xi)$ of (1-7) appropriately, the operator $L_{4}$ belongs to $A(m)$ but does not necessarily satisfy (1-8).

Generally speaking, the operator $L u$ defined by (1-3) may meanwhile belong to several classes denoted by $A\left(m_{1}\right), \ldots, A\left(m_{k}\right)$, where $m_{1} \leq m_{2} \leq \cdots \leq m_{k}$. For example, the operators of $L_{2}, L_{3}$ belong to both $A(m-1)$ and $A(m)$. Throughout the paper, when we say that $L$ belongs to the class of $A(m)$, we always mean $m$ is the largest value $m_{k}$.

The purpose of this paper is to provide a very simple geometric condition of volume growth on $M^{N}$ to suffice that the only nonnegative solution $u$ of (1-1) is identically zero. Let us emphasize that there is no curvature assumption on manifolds throughout the paper.

First, let us give our setting on manifolds. Let $M^{N}$ be a connected geodesically complete noncompact Riemannian manifold. Denote by $\mu$ the Riemannian measure, and by $B(x, r)$ the geodesic ball on $M^{N}$ of radius $r$ centered at $x \in M^{N}$. Given that $d(\cdot, \cdot)$ is the geodesic distance and that $x_{0}$ is a reference point on $M$, define $B_{r}:=B\left(x_{0}, r\right)$ for simplicity, where $r=d\left(x, x_{0}\right)$. Assume also throughout the paper that $V(x) \in L_{\mathrm{loc}}^{\infty}\left(M^{N}\right)$.

The problem of investigating the uniqueness of nonnegative solutions has attracted a lot of attention, especially in the Euclidean space. For example, if $M^{N}=\mathbb{R}^{N}$ with $N \geq 2$, in the case of $V(x) \equiv 1$, the problem (1-1) was systematically investigated by 
Kurta [1999]. By using the nonlinear capacity arguments, he obtained nonexistence results concerning different differential inequalities. For a specific operator $L$, let us recommend a series of papers of Mitidieri and Pokhozhaev [1998; 1999; 2001] for a more comprehensive description. Related problems have also been studied in massive literatures; see [Caristi et al. 2008; Caristi and Mitidieri 1997; D'Ambrosio 2009; D'Ambrosio and Mitidieri 2010; Ni and Serrin 1985; 1986] and the references therein.

Let us turn to the results in the Riemannian manifolds setting. The celebrated idea of studying the uniqueness of nonnegative solutions in terms of the volume of the geodesic ball was due to Cheng and Yau [1975]. They obtained the following marvelous result: if the volume estimate

$$
\mu\left(B_{r}\right) \leq C r^{2}
$$

holds for all large enough $r$, then any positive solution to $\Delta u \leq 0$ is identically constant.

The amazing point of Cheng and Yau's result is that there is no assumption on either curvature or the behavior of the solution near infinity, only in terms of volume growth.

Very recently, this idea was used and developed in [Grigor'yan and Kondratiev 2010; Grigor'yan and Sun 2014; Sun 2014] to investigate the differential inequality of the form

$$
\operatorname{div}(A(x) \nabla u)+V(x) u^{\sigma} \leq 0,
$$

where $\sigma>1$. Particularly, when $A(x)=$ Id and $V(x)=1$, (1-9) becomes

$$
\Delta u+u^{\sigma} \leq 0 .
$$

In [Grigor'yan and Sun 2014] it is proved that if

$$
\mu\left(B_{r}\right) \leq C r^{\frac{2 \sigma}{\sigma-1}} \ln ^{\frac{1}{\sigma-1}} r
$$

holds for all large enough $r$, then the only nonnegative solution of (1-10) is identically zero. Moreover, the exponents $2 \sigma /(\sigma-1)$ and $1 /(\sigma-1)$ are sharp and cannot be relaxed.

Let us define the weak nonnegative solution of (1-1). For convenience, we introduce the notation

$$
A_{u}=(A(x, u, \nabla u), \nabla u)
$$

and

$$
W_{\mathrm{loc}}^{1, m}\left(M^{N}\right):=\left\{f \mid f \in L_{\mathrm{loc}}^{m}\left(M^{N}\right), \nabla f \in L_{\mathrm{loc}}^{m}\left(M^{N}\right)\right\},
$$


and denote by $W_{c}^{1, m}\left(M^{N}\right)$ the subspace of $W_{\text {loc }}^{1, m}\left(M^{N}\right)$ of functions with compact support.

Definition 1.1. A function $u$ on $M^{N}$ is called a weak nonnegative solution of (1-1) if $u \in W_{\mathrm{loc}}^{1, m}\left(M^{N}\right)$ and $A_{u} \in L_{\mathrm{loc}}^{1}\left(M^{N}\right)$ and if, for any nonnegative function $\psi \in W_{c}^{1, m}\left(M^{N}\right)$, the following inequality holds:

$$
-\int_{M^{N}}(A(x, u, \nabla u), \nabla \psi) d \mu+\int_{M^{N}} V(x) u^{\sigma} \psi d \mu \leq 0,
$$

where $(\cdot, \cdot)$ is the inner product in $T_{x}\left(M^{N}\right)$ given by a Riemannian metric.

Remark 1.2. If $u$ is a weak nonnegative solution of (1-1), and the operator $L$ belongs to the class $A(m)$, we know

$$
\begin{aligned}
\int_{M^{N}}(A(x, u, \nabla u), \nabla \psi) d \mu & \leq C \int_{M^{N}}|\nabla \psi| A_{u}^{\frac{m-1}{m}} d \mu \\
& \leq C\left(\int_{M^{N}}|\nabla \psi|^{m} d \mu\right)^{\frac{1}{m}}\left(\int_{\operatorname{supp}(\psi)} A_{u} d \mu\right)^{\frac{m-1}{m}}<\infty .
\end{aligned}
$$

Hence, by the definition of the solution, we know the second integral in (1-13) is bounded.

Define

$$
p=\frac{m \sigma}{\sigma-m+1}, \quad q=\frac{m-1}{\sigma-m+1},
$$

and introduce a new measure $v$ defined by

$$
d \nu=V^{-\frac{m-1}{\sigma-m+1}} d \mu .
$$

Assume that $V$ satisfies the following condition: for some nonnegative constants $\delta_{1}, \delta_{2}$, the estimate

$$
c r^{-\delta_{1}} \leq V(x) \leq C r^{\delta_{2}}
$$

holds for all large enough $r$.

Theorem 1.3. Assume that operator $L$ in (1-1) belongs to the class of $A(m)$ with $1<m<\sigma+1$. Assume also that (V) holds with $\delta_{1}, \delta_{2} \geq 0$. If the inequality

$$
v\left(B_{r} \backslash \overline{B_{1}}\right) \leq C r^{p} \ln ^{q} r
$$

holds for all large enough $r$, then the only nonnegative solution of (1-1) is identically zero.

Remark 1.4. It is not clear that the sharpness of exponents $p$ and $q$ in (1-16) holds for all the operators of the class $A(m)$. However, in many specific cases, the exponents $p, q$ are sharp; one can refer to [Grigor'yan and Sun 2014; Sun 2014; 2015]. 
Notation. The letters $C, C^{\prime}, C_{0}, C_{1}, \ldots$ denote positive constants whose values are unimportant and may vary at different occurrences.

In Section 2, we show the proof of Theorem 1.3. In Section 3, we present two examples to show that our result is very inclusive.

\section{Proof of Theorem 1.3}

Let $u$ be a nonnegative solution of (1-1). Fix some ball $B_{R}$, where $R>0$ is to be chosen later. Take a Lipschitz function $\varphi$ on $M^{N}$ with compact support, such that $0 \leq \varphi \leq 1$ and $\varphi \equiv 1$ in a neighborhood of $\overline{B_{R}}$. Particularly, $\varphi \in W_{c}^{1, m}\left(M^{N}\right)$. We use the following test function for (1-13):

$$
\psi_{\rho}(x)=\varphi(x)^{s}(u+\rho)^{-t}
$$

where $\rho>0$ is a parameter near zero, and $s$ will be chosen to be a large enough fixed constant, and $t$ will take arbitrarily small positive values near zero.

Since $1 /(u+\rho)$ is bounded, $\psi_{\rho}$ has compact support and is bounded. The identity

$$
\nabla \psi_{\rho}=-t \varphi^{s}(u+\rho)^{-t-1} \nabla u+s \varphi^{s-1}(u+\rho)^{-t} \nabla \varphi
$$

implies that $\nabla \psi_{\rho} \in L^{m}\left(M^{N}\right)$, hence, $\psi_{\rho} \in W_{c}^{1, m}\left(M^{N}\right)$. We obtain from (1-13) that

$$
\begin{aligned}
t \int_{M^{N}} \varphi^{s}(u+\rho)^{-t-1} A_{u} d \mu & +\int_{M^{N}} \varphi^{s} V u^{\sigma}(u+\rho)^{-t} d \mu \\
& \leq s \int_{M^{N}} \varphi^{s-1}(u+\rho)^{-t}(A(x, u, \nabla u), \nabla \varphi) d \mu .
\end{aligned}
$$

Estimate the right-hand side of (2-2) by the Young inequality

$$
\int_{M^{N}} f g d \mu \leq \epsilon \int_{M^{N}}|f|^{p_{0}} d \mu+C_{\epsilon} \int_{M^{N}}|g|^{p_{0}^{\prime}} d \mu,
$$

where $1 / p_{0}+1 / p_{0}^{\prime}=1$. Letting $p_{0}=m /(m-1)$, and using (1-3), we obtain

$$
\begin{aligned}
& s \int_{M^{N}} \varphi^{s-1}(u+\rho)^{-t}(A(x, u, \nabla u), \nabla \varphi) d \mu \\
& \leq C s \int_{M^{N}} \varphi^{s-1}(u+\rho)^{-t} A_{u}^{\frac{m-1}{m}}|\nabla \varphi| d \mu \\
& =C \int_{M^{N}}\left[t^{\frac{1}{p_{0}}} \varphi^{\frac{s}{p_{0}}}(u+\rho)^{-\frac{t+1}{p_{0}}} A_{u}^{\frac{m-1}{m}}\right]\left[\frac{s}{t^{p_{0}}} \varphi^{\frac{s}{p_{0}^{\prime}}-1}(u+\rho)^{1-\frac{t+1}{p_{0}^{\prime}}}|\nabla \varphi|\right] d \mu \\
& \leq \frac{t}{2} \int_{M^{N}} \varphi^{s}(u+\rho)^{-t-1} A_{u} d \mu+C \frac{s^{m}}{t^{m-1}} \int_{M^{N}} \varphi^{s-m}(u+\rho)^{m-t-1}|\nabla \varphi|^{m} d \mu .
\end{aligned}
$$


Substituting the above into (2-2), and canceling out half of the first term in (2-2), we obtain

$$
\begin{aligned}
\frac{t}{2} \int_{M^{N}} \varphi^{s}(u+\rho)^{-t-1} A_{u} d \mu & +\int_{M^{N}} \varphi^{s} V u^{\sigma}(u+\rho)^{-t} d \mu \\
& \leq C \frac{s^{m}}{t^{m-1}} \int_{M^{N}} \varphi^{s-m}(u+\rho)^{m-t-1}|\nabla \varphi|^{m} d \mu .
\end{aligned}
$$

Using the Young inequality again in the right-hand side of (2-4) with

$$
p_{1}=\frac{\sigma-t}{m-t-1}, \quad p_{1}^{\prime}=\frac{\sigma-t}{\sigma-m+1},
$$

we obtain

$$
\begin{aligned}
& \frac{s^{m}}{t^{m-1}} \int_{M^{N}} \varphi^{s-m}(u+\rho)^{m-t-1}|\nabla \varphi|^{m} d \mu \\
& =\int_{M^{N}}\left[\varphi^{\frac{s}{p_{1}}} V^{\frac{1}{p_{1}}}(u+\rho)^{\frac{\sigma-t}{p_{1}}}\right]\left[\frac{s^{m}}{t^{m-1}} \varphi^{\frac{s}{p_{1}^{\prime}}-m} V^{-\frac{1}{p_{1}}}|\nabla \varphi|^{m}\right] d \mu \\
& \leq \frac{1}{2} \int_{M^{N}} \varphi^{s} V(u+\rho)^{\sigma-t} d \mu \\
& \quad+C\left(\frac{s^{m}}{t^{m-1}}\right)^{\frac{\sigma-t}{\sigma-m+1}} \int_{M^{N}} \varphi^{s-\frac{m(\sigma-t)}{\sigma-m+1}} V^{-\frac{m-t-1}{\sigma-m+1}}|\nabla \varphi|^{\frac{m(\sigma-t)}{\sigma-m+1}} d \mu .
\end{aligned}
$$

Using in the right-hand side of (2-5) the simple inequality

$$
\left(\frac{s^{m}}{t^{m-1}}\right)^{\frac{\sigma-t}{\sigma-m+1}} \leq\left(\frac{s^{m}}{t^{m-1}}\right)^{\frac{\sigma}{\sigma-m+1}}
$$

and combining (2-5) with (2-4), we obtain that

$$
\begin{aligned}
& \frac{t}{2} \int_{M^{N}} \varphi^{s}(u+\rho)^{-t-1} A_{u} d \mu+\int_{M^{N}} \varphi^{s} V u^{\sigma}(u+\rho)^{-t} d \mu \\
& \leq \frac{1}{2} \int_{M^{N}} \varphi^{s} V(u+\rho)^{\sigma-t} d \mu \\
& \quad+C t^{-\frac{\sigma(m-1)}{\sigma-m+1}} \int_{M^{N}} \varphi^{s-\frac{m(\sigma-t)}{\sigma-m+1}} V^{-\frac{m-t-1}{\sigma-m+1}}|\nabla \varphi|^{\frac{m(\sigma-t)}{\sigma-m+1}} d \mu,
\end{aligned}
$$

where the value of $s$ is absorbed into the constant $C$.

It is easy to obtain from the definition of the solution the boundedness of the term

$$
\int_{M^{N}} \varphi^{s} V u^{\sigma}(u+\rho)^{-t} d \mu .
$$

Then the boundedness of $\int_{M^{N}} \varphi^{s} V(u+\rho)^{\sigma-t} d \mu$ follows by the boundedness of

$$
\int_{M^{N}} \varphi^{s} V u^{\sigma}(u+\rho)^{-t} d \mu,
$$


and by the fact that $V \in L_{\mathrm{loc}}^{1}\left(M^{N}\right)$.

By the dominated convergence theorem, we know

$$
\lim _{\rho \downarrow 0} \int_{M^{N}} \varphi^{s} V(u+\rho)^{\sigma-t} d \mu=\int_{M^{N}} \varphi^{s} V u^{\sigma-t} d \mu .
$$

Letting $\rho \downarrow 0$ in (2-6) and applying the monotone convergence theorem, we have

$$
\begin{aligned}
\frac{t}{2} \int_{M^{N}} \varphi^{s} u^{-t-1} A_{u} d \mu+\int_{M^{N}} \varphi^{s} V u^{\sigma-t} d \mu \\
\quad \leq \frac{1}{2} \int_{M^{N}} \varphi^{s} V u^{\sigma-t} d \mu+C t^{-\frac{\sigma(m-1)}{\sigma-m+1}} \int_{M^{N}} \varphi^{s-\frac{m(\sigma-t)}{\sigma-m+1}} V^{-\frac{m-t-1}{\sigma-m+1}}|\nabla \varphi|^{\frac{m(\sigma-t)}{\sigma-m+1}} d \mu,
\end{aligned}
$$

which is

$$
\begin{aligned}
\frac{t}{2} \int_{M^{N}} \varphi^{s} u^{-t-1} A_{u} d \mu+ & \frac{1}{2} \int_{M^{N}} \varphi^{s} V u^{\sigma-t} d \mu \\
& \leq C t^{-\frac{\sigma(m-1)}{\sigma-m+1}} \int_{M^{N}} \varphi^{s-\frac{m(\sigma-t)}{\sigma-m+1}} V^{-\frac{m-t-1}{\sigma-m+1}}|\nabla \varphi|^{\frac{m(\sigma-t)}{\sigma-m+1}} d \mu .
\end{aligned}
$$

Applying (1-13) once more, using another test function $\psi=\varphi^{s}$, we obtain

$$
\begin{array}{rl}
\int_{M^{N}} \varphi^{s} & V u^{\sigma} d \mu \\
& \leq s \int_{M^{N}} \varphi^{s-1}(A(x, u, \nabla u), \nabla \varphi) d \mu \\
& \leq C s \int_{M^{N}} \varphi^{s-1} A_{u}^{\frac{m-1}{m}}|\nabla \varphi| d \mu \\
& \leq C s\left(\int_{M^{N}} \varphi^{s} u^{-t-1} A_{u} d \mu\right)^{\frac{m-1}{m}}\left(\int_{M^{N}} \varphi^{s-m} u^{(t+1)(m-1)}|\nabla \varphi|^{m} d \mu\right)^{\frac{1}{m}} .
\end{array}
$$

From (2-7), we obtain

$$
\int_{M^{N}} \varphi^{s} u^{-t-1} A_{u} d \mu \leq C t^{-1-\frac{\sigma(m-1)}{\sigma-m+1}} \int_{M^{N}} \varphi^{s-\frac{m(\sigma-t)}{\sigma-m+1}} V^{-\frac{m-t-1}{\sigma-m+1}}|\nabla \varphi|^{\frac{m(\sigma-t)}{\sigma-m+1}} d \mu .
$$

Substituting into (2-8) yields

$$
\begin{aligned}
\int_{M^{N}} \varphi^{s} V u^{\sigma} d \mu \leq C\left[t^{-1-\frac{\sigma(m-1)}{\sigma-m+1}} \int_{M^{N}} \varphi^{s-\frac{m(\sigma-t)}{\sigma-m+1}} V^{-\frac{m-t-1}{\sigma-m+1}}|\nabla \varphi|^{\frac{m(\sigma-t)}{\sigma-m+1}} d \mu\right]^{\frac{m-1}{m}} \\
\times\left[\int_{M^{N}} \varphi^{s-m} u^{(t+1)(m-1)}|\nabla \varphi|^{m} d \mu\right]^{\frac{1}{m}} .
\end{aligned}
$$

Recalling that $\nabla \varphi=0$ on $B_{R}$ and applying the Hölder inequality to the last term of (2-9) with the Hölder couple

$$
p_{2}=\frac{\sigma}{(t+1)(m-1)}, \quad p_{2}^{\prime}=\frac{\sigma}{\sigma-(t+1)(m-1)},
$$


we obtain

$$
\begin{aligned}
\int_{M^{N}} & \varphi^{s-m} u^{(t+1)(m-1)}|\nabla \varphi|^{m} d \mu \\
= & \int_{M^{N} \backslash B_{R}}\left(\varphi^{\frac{s}{p_{2}}} V^{\frac{1}{p_{2}}} u^{(t+1)(m-1)}\right)\left(\varphi^{\frac{s}{p_{2}^{\prime}}-m} V^{-\frac{1}{p_{2}}}|\nabla \varphi|^{m}\right) d \mu \\
\leq & \left(\int_{M^{N} \backslash B_{R}} \varphi^{s} V u^{\sigma} d \mu\right)^{\frac{(t+1)(m-1)}{\sigma}} \\
& \times\left(\int_{M^{N} \backslash B_{R}} \varphi^{s-\frac{m \sigma}{\sigma-(t+1)(m-1)}} V^{-\frac{(t+1)(m-1)}{\sigma-(t+1)(m-1)}}|\nabla \varphi|^{\frac{m \sigma}{\sigma-(t+1)(m-1)}} d \mu\right)^{\frac{\sigma-(t+1)(m-1)}{\sigma}} .
\end{aligned}
$$

Substituting (2-10) into (2-9), choosing $s$ large enough, and noting that $\varphi \leq 1$, we obtain

$$
\begin{aligned}
& \int_{M^{N}} \varphi^{s} V u^{\sigma} d \mu \leq C t^{-\frac{m-1}{m}}-\frac{\sigma(m-1)^{2}}{m(\sigma-m+1)}\left(\int_{M^{N}} V^{-\frac{m-1-t}{\sigma-m+1}}|\nabla \varphi|^{\frac{m(\sigma-t)}{\sigma-m+1}} d \mu\right)^{\frac{m-1}{m}} \\
& \times\left(\int_{M^{N}} V^{-\frac{(t+1)(m-1)}{\sigma-(t+1)(m-1)}}|\nabla \varphi|^{\frac{m \sigma}{\sigma-(t+1)(m-1)}} d \mu\right)^{\frac{\sigma-(t+1)(m-1)}{m \sigma}} \\
& \times\left(\int_{M^{N} \backslash B_{R}} \varphi^{S} V u^{\sigma} d \mu\right)^{\frac{(t+1)(m-1)}{m \sigma}} .
\end{aligned}
$$

From the definition of the solution, we know $\int_{M^{N}} \varphi^{s} V u^{\sigma} d \mu$ is finite. It follows from (2-11) that

$$
\begin{gathered}
\left(\int_{M^{N}} \varphi^{s} V u^{\sigma} d \mu\right)^{1-\frac{(t+1)(m-1)}{m \sigma}} \\
\leq C t^{-\frac{m-1}{m}-\frac{\sigma(m-1)^{2}}{m(\sigma-m+1)}}\left(\int_{M^{N}} V^{-\frac{m-1-t}{\sigma-m+1}}|\nabla \varphi|^{\frac{m(\sigma-t)}{\sigma-m+1}} d \mu\right)^{\frac{m-1}{m}} \\
\quad \times\left(\int_{M^{N}} V^{-\frac{(t+1)(m-1)}{\sigma-(t+1)(m-1)}}|\nabla \varphi|^{\frac{m \sigma}{\sigma-(t+1)(m-1)}} d \mu\right)^{\frac{\sigma-(t+1)(m-1)}{m \sigma}}
\end{gathered}
$$

Note that the first integral in the right-hand side of (2-12) has the estimate

$$
\int_{M^{N}} V^{-\frac{m-1-t}{\sigma-m+1}}|\nabla \varphi|^{\frac{m(\sigma-t)}{\sigma-m+1}} d \mu \leq \int_{M^{N}}|\nabla \varphi|^{\frac{m(\sigma-t)}{\sigma-m+1}} V^{\frac{t}{\sigma-m+1}} d \nu
$$

where we have used that $d v=V^{-\frac{m-1}{\sigma-m+1}} d \mu$. Similarly, the second integral in the right-hand side of (2-12) can be estimated as follows:

$$
\begin{aligned}
\int_{M^{N}} V^{-\frac{(t+1)(m-1)}{\sigma-(t+1)(m-1)}}|\nabla \varphi|^{\frac{m \sigma}{\sigma-(t+1)(m-1)}} d \mu & \\
& \leq \int_{M^{N}}|\nabla \varphi|^{\frac{m \sigma}{\sigma-(t+1)(m-1)}} V^{-\frac{t \sigma(m-1)}{[\sigma-(t+1)(m-1)](\sigma-m+1)}} d \nu .
\end{aligned}
$$


Substituting (2-13) and (2-14) into (2-11), we have

$$
\begin{aligned}
& \int_{M^{N}} \varphi^{S} V u^{\sigma} d \mu \\
& \leq C t^{-\frac{m-1}{m}-\frac{\sigma(m-1)^{2}}{m(\sigma-m+1)}}\left(\int_{M^{N}}|\nabla \varphi|^{\frac{m(\sigma-t)}{\sigma-m+1}} V^{\frac{t}{\sigma-m+1}} d \nu\right)^{\frac{m-1}{m}} \\
& \times\left(\int_{M^{N}}|\nabla \varphi|^{\frac{m \sigma}{\sigma-(t+1)(m-1)}} V^{-\frac{t \sigma(m-1)}{[\sigma-(t+1)(m-1)(\sigma-m+1)}} d \nu\right)^{\frac{\sigma-(t+1)(m-1)}{m \sigma}} \\
& \times\left(\int_{M^{N} \backslash B_{R}} \varphi^{S} V u^{\sigma} d \mu\right)^{\frac{(t+1)(m-1)}{m \sigma}} .
\end{aligned}
$$

Substituting (2-13) and (2-14) into (2-12), we obtain

$$
\begin{aligned}
& \left(\int_{M^{N}} \varphi^{s} V u^{\sigma} d \mu\right)^{1-\frac{(t+1)(m-1)}{m \sigma}} \\
& \leq C t^{-\frac{m-1}{m}-\frac{\sigma(m-1)^{2}}{m(\sigma-m+1)}}\left(\int_{M^{N}}|\nabla \varphi|^{\frac{m(\sigma-t)}{\sigma-m+1}} V^{\frac{t}{\sigma-m+1}} d \nu\right)^{\frac{m-1}{m}} \\
& \quad \times\left(\int_{M^{N}}|\nabla \varphi|^{\frac{m \sigma}{\sigma-(t+1)(m-1)}} V^{-\frac{t \sigma(m-1)}{[\sigma-(t+1)(m-1)](\sigma-m+1)}} d \nu\right)^{\frac{\sigma-(t+1)(m-1)}{m \sigma}} .
\end{aligned}
$$

Let $\left\{\tilde{\varphi}_{k}\right\}_{k \in \mathbb{N}}$ be a sequence for which each $\tilde{\varphi}_{k}$ is a Lipschitz function such that $\operatorname{supp}\left(\tilde{\varphi}_{k}\right) \subset B_{2^{k}}$, and $\tilde{\varphi}_{k}=1$ in a neighborhood of $B_{2^{k-1}}$, and

$$
\left|\nabla \tilde{\varphi}_{k}\right| \begin{cases}\leq \frac{C}{2^{k-1}} & \text { for } x \in B_{2^{k}} \backslash B_{2^{k-1}}, \\ =0 & \text { otherwise, }\end{cases}
$$

where $C$ does not depend on $k$.

Fix some $n \in \mathbb{N}$ and set

$$
t=\frac{1}{n}
$$

and

$$
\varphi_{n}=\frac{\sum_{k=n+1}^{2 n} \tilde{\varphi}_{k}}{n}
$$

Note that $\varphi_{n}=1$ on $B_{2^{n}}$, and $\varphi_{n}=0$ outside $B_{2^{2 n}}$, and $0 \leq \varphi_{n} \leq 1$ on $M^{N}$. Note that, for any $a \geq 1$, using that $\operatorname{supp}\left(\nabla \tilde{\varphi}_{k}\right)$ are disjoint, we have

$$
\left|\nabla \varphi_{n}\right|^{a}=\frac{\sum_{k=n+1}^{2 n}\left|\nabla \tilde{\varphi}_{k}\right|^{a}}{n^{a}} .
$$

It is easy to see that

$$
\varphi_{n} \in W_{\mathrm{loc}}^{1, m}\left(M^{N}\right)
$$


Consider the integral

$$
J_{n}(a, b)=\int_{M^{N}}\left|\nabla \varphi_{n}\right|^{a} V^{b} d \mu,
$$

where $a, b$ are taking values from

$$
(a, b)=\left\{\begin{array}{l}
\left(\frac{m(\sigma-t)}{\sigma-m+1}, \frac{t}{\sigma-m+1}\right), \\
\left(\frac{m \sigma}{\sigma-(t+1)(m-1)},-\frac{t \sigma(m-1)}{[\sigma-(t+1)(m-1)](\sigma-m+1)}\right) .
\end{array}\right.
$$

We write $a$ in the form

$$
a=p+l t
$$

with the corresponding two values of $l$,

$$
l_{1}=-\frac{m}{\sigma-m+1}, \quad l_{2}=\frac{m \sigma(m-1)}{[\sigma-(t+1)(m-1)](\sigma-m+1)},
$$

where $p=m \sigma /(\sigma-m+1)$.

For $b \geq 0$, we know

$$
\begin{aligned}
J_{n}(a, b) & =\int_{M^{N}}\left|\nabla \varphi_{n}\right|^{a} V^{b} d v \\
& =\int_{M^{N}} \frac{\sum_{k=n+1}^{2 n}\left|\nabla \tilde{\varphi}_{k}\right|^{a}}{n^{a}} V^{b} d v \\
& \leq \sum_{k=n+1}^{2 n} \int_{B_{2^{k}} \backslash B_{2^{k-1}}} \frac{\left|\nabla \tilde{\varphi}_{k}\right|^{a}}{n^{a}} V^{b} d v \\
& \leq C \sum_{k=n+1}^{2 n} \int_{B_{2^{k}} \backslash B_{2^{k-1}}}\left(\frac{2^{1-k}}{n}\right)^{a} r^{\delta_{2} b} d v \\
& \leq C \sum_{k=n+1}^{2 n}\left(\frac{2^{1-k}}{n}\right)^{a}\left(2^{k}\right)^{\delta_{2} b} v\left(B_{2^{k}} \backslash B_{1}\right)
\end{aligned}
$$

Note that $a=p+l t$, and $n+1 \leq k \leq 2 n$, and

$$
\begin{aligned}
\left(\frac{2^{1-k}}{n}\right)^{a}\left(2^{k}\right)^{\delta_{2} b} & =\left(\frac{2^{-k}}{n}\right)^{p}\left(\frac{2^{-k}}{n}\right)^{l t}\left(2^{k}\right)^{\delta_{2} b} \\
& \leq\left(\frac{2^{-k}}{n}\right)^{p}\left(2^{k}\right)^{\delta_{2} b} \sup _{n+1 \leq k \leq 2 n}\left(\frac{2^{-k}}{n}\right)^{l t} \\
& \leq C\left(\frac{2^{-k}}{n}\right)^{p}\left(2^{k}\right)^{\delta_{2} b} .
\end{aligned}
$$


Substituting (2-26) into (2-25), and using the volume growth (1-16), we obtain

$$
\begin{aligned}
J_{n}(a, b) & \leq C \sum_{k=n+1}^{2 n}\left(\frac{2^{-k}}{n}\right)^{p}\left(2^{k}\right)^{\delta_{2} b} v\left(B_{2^{k}} \backslash B_{1}\right) \\
& \leq C \sum_{k=n+1}^{2 n}\left(\frac{2^{-k}}{n}\right)^{p}\left(2^{k}\right)^{\delta_{2} b}\left(2^{k}\right)^{p} \ln ^{q}\left(2^{k}\right) \\
& \leq C \frac{1}{n^{p}} \sum_{k=n+1}^{2 n} k^{q} 2^{k \delta_{2} b} \leq C n^{q+1-p} 2^{2 n \delta_{2} b} \leq C n^{-\frac{\sigma(m-1)}{\sigma-m+1}} 2^{2 n \delta_{2} b} .
\end{aligned}
$$

Similarly, for the case of $b \leq 0$, we obtain

$$
J_{n}(a, b) \leq C n^{-\frac{\sigma(m-1)}{\sigma-m+1}} 2^{-2 n \delta_{1} b} .
$$

Taking the sequence $\left\{\varphi_{n}\right\}$ in (2-16), we obtain

$$
\begin{aligned}
& \left(\int_{M^{N}} \varphi_{n}^{S} V u^{\sigma} d \mu\right)^{1-\frac{(t+1)(m-1)}{m \sigma}} \\
& \leq C t^{-\frac{m-1}{m}-\frac{\sigma(m-1)^{2}}{m(\sigma-m+1)}}\left(J_{n}\left(\frac{m(\sigma-t)}{\sigma-m+1}, \frac{t}{\sigma-m+1}\right)\right)^{\frac{m-1}{m}} \\
& \quad \times\left(J_{n}\left(\frac{m \sigma}{\sigma-(t+1)(m-1)}, \frac{-t \sigma(m-1)}{[\sigma-(t+1)(m-1)](\sigma-m+1)}\right)\right)^{\frac{\sigma-(t+1)(m-1)}{m \sigma}} .
\end{aligned}
$$

Substituting (2-27) and (2-28) and noting that $t=1 / n$, we obtain

$$
\begin{aligned}
\left(\int_{M^{N}} \varphi_{n}^{S} V u^{\sigma} d \mu\right)^{1-\frac{\left(\frac{1}{n}+1\right)(m-1)}{m \sigma}} & \\
\leq & C n^{\frac{m-1}{m}+\frac{\sigma(m-1)^{2}}{m(\sigma-m+1)}}\left(n^{-\frac{\sigma(m-1)}{\sigma-m+1}} 2^{2 n \delta_{2} \frac{1}{\sigma-m+1}}\right)^{\frac{m-1}{m}} \\
& \times\left(n^{-\frac{\sigma(m-1)}{\sigma-m+1}} 2^{2 n \delta_{1} \frac{\frac{1}{n} \sigma(m-1)}{\left[\sigma-\left(\frac{1}{n}+1\right)(m-1)\right](\sigma-m+1)}}\right)^{\frac{\sigma-\left(\frac{1}{n}+1\right)(m-1)}{m \sigma}} \\
\leq & C n^{\frac{(m-1)^{2}}{n(\sigma-m+1)}} 2^{\frac{2\left(\delta_{1}+\delta_{2}\right)(m-1)}{m(\sigma-m+1)}} .
\end{aligned}
$$

Noting that $\varphi_{n}=1$ on $B_{2^{n}}$ and taking the lim sup of both sides in (2-30) as $n \rightarrow \infty$, we obtain

$$
\int_{M^{N}} V u^{\sigma} d \mu \leq C<\infty
$$


Applying similar arguments to (2-15), we obtain that

$$
\int_{M^{N}} \varphi_{n}^{s} V u^{\sigma} d \mu \leq C\left(\int_{M^{N} \backslash B_{2^{n}}} \varphi_{n}^{s} V u^{\sigma} d \mu\right)^{\frac{\left(\frac{1}{n}+1\right)(m-1)}{m \sigma}} .
$$

Since $\varphi_{n}=1$ on $B_{2^{n}}$, we have

$$
\int_{B_{2^{n}}} V u^{\sigma} d \mu \leq C\left(\int_{M^{N} \backslash B_{2^{n}}} \varphi_{n}^{s} V u^{\sigma} d \mu\right)^{\frac{\left(\frac{1}{n}+1\right)(m-1)}{m \sigma}} .
$$

Combining this with (2-31) and letting $n \rightarrow \infty$, we obtain that

$$
\int_{M^{N}} V u^{\sigma} d \mu=0
$$

since $V>0$ for almost all $x \in M^{N}$. Thus $u \equiv 0$.

\section{Examples}

Our result can cover many known results in the case of $M^{N}=R^{N}$. Let us mention two of these examples.

Example 1. Let us investigate the inequality

$$
\operatorname{div}\left(|\nabla u|^{m-2} \nabla u\right)+V(x) u^{\sigma} \leq 0, \quad \text { in } \mathbb{R}^{N},
$$

where $V(x)=1 /|x|^{\gamma}$ for $|x| \geq 1$, and $N>m>\max \{1, \gamma\}$, and $\sigma>m-1$.

By [Filippucci 2009, Corollary 1.5], we know if

$$
\sigma \leq \frac{(N-\gamma)(m-1)}{N-m},
$$

then (3-1) has no positive solutions in some natural class. Compared to our result of Theorem 1.3, we know for large $r$

$$
v\left(B_{r} \backslash \overline{B_{1}}\right)=\int_{B_{r} \backslash \overline{B_{1}}} V^{-\frac{m-1}{\sigma-m+1}} d \mu=\omega_{N} \int_{1}^{r} s^{\frac{\gamma(m-1)}{\sigma-m+1}} s^{N-1} d s \approx C r^{N+\frac{\gamma(m-1)}{\sigma-m+1}},
$$

where $\omega_{N}$ is the surface area of the unit ball in $\mathbb{R}^{N}$, and $\mu$ is the Lebesgue measure, and the sign $\approx$ means that both the inequalities $\leq$ and $\geq$ are satisfied but with different values of different constants $c, C$.

By (3-3), it follows that the condition (1-16) is equivalent to

$$
N+\frac{\gamma(m-1)}{\sigma-m+1} \leq p=\frac{m \sigma}{\sigma-m+1},
$$

which in turn is equivalent to (3-2). 
Example 2. Consider the differential inequality

$$
\operatorname{div}\left(\frac{\nabla u}{\sqrt{1+|\nabla u|^{2}}}\right)+u^{\sigma} \leq 0, \quad \text { in } \mathbb{R}^{N},
$$

where $N>2, \sigma>1$. This problem was investigated in [Mitidieri and Pokhozhaev 1999]. They obtained that if

$$
\sigma \leq \frac{N}{N-2}
$$

then (3-5) has no positive solutions. Note that the operator in (3-5) belongs to the class of $A(2)$, and that $v\left(B_{r} \backslash \overline{B_{1}}\right)=\mu\left(B_{r} \backslash \overline{B_{1}}\right) \approx C r^{N}$. By Theorem 1.3, we know if

$$
N \leq \frac{2 \sigma}{\sigma-1}
$$

then (3-5) has no positive solution. It is easy to check that (3-6) and (3-7) are equivalent.

\section{Acknowledgements}

The author would like to express his gratitude to the International Graduate College of Bielefeld University for the financial support. The author is also grateful to the anonymous referee for careful reading and valuable suggestions which helped improve the quality of the paper.

\section{References}

[Caristi and Mitidieri 1997] G. Caristi and E. Mitidieri, "Nonexistence of positive solutions of quasilinear equations", Adv. Differential Equations 2:3 (1997), 319-359. MR 98a:34011 Zbl 1023.34500

[Caristi et al. 2008] G. Caristi, L. D'Ambrosio, and E. Mitidieri, "Liouville theorems for some nonlinear inequalities", Proc. Steklov Inst. Math. 260 (2008), 90-111. In Russian; translated in Tr. Mat. Inst. Steklova 260 (2008), 97-118. Zbl 1233.35207

[Cheng and Yau 1975] S. Y. Cheng and S. T. Yau, "Differential equations on Riemannian manifolds and their geometric applications", Comm. Pure Appl. Math. 28:3 (1975), 333-354. MR 52 \#6608 Zbl 0312.53031

[D'Ambrosio 2009] L. D'Ambrosio, "Liouville theorems for anisotropic quasilinear inequalities", Nonlinear Anal. 70:8 (2009), 2855-2869. MR 2010e:35097 Zbl 1177.35262

[D'Ambrosio and Mitidieri 2010] L. D'Ambrosio and E. Mitidieri, “A priori estimates, positivity results, and nonexistence theorems for quasilinear degenerate elliptic inequalities", Adv. Math. 224:3 (2010), 967-1020. MR 2011e:35109 Zbl 1206.35265

[Filippucci 2009] R. Filippucci, "Nonexistence of positive weak solutions of elliptic inequalities", Nonlinear Anal. 70:8 (2009), 2903-2916. MR 2010f:35450 Zbl 1165.35487

[Grigor'yan and Kondratiev 2010] A. Grigor'yan and V. A. Kondratiev, "On the existence of positive solutions of semilinear elliptic inequalities on Riemannian manifolds", pp. 203-218 in Around the research of Vladimir Maz'ya, II, edited by A. Laptev, Int. Math. Ser. (N. Y.) 12, Springer, New York, 2010. MR 2011m:58030 Zbl 1185.35344 
[Grigor'yan and Sun 2014] A. Grigor'yan and Y. Sun, "On nonnegative solutions of the inequality $\Delta u+u^{\sigma} \leq 0$ on Riemannian manifolds", Comm. Pure Appl. Math. 67:8 (2014), 1336-1352. MR 3225632 Zbl 1296.58011

[Kurta 1999] V. V. Kurta, "The nonexistence of positive solutions to some elliptic equations", Mat. Zametki 65:4 (1999), 552-561. In Russian; translated in Math. Notes 65:4 (1999), 462-469. MR 2000f:35045 Zbl 0964.35043

[Mīkljukov 1979] V. M. Mīkljukov, "A new approach to the Bernšteĭn theorem and to related questions of equations of minimal surface type”, Mat. Sb. (N.S.) 108(150):2 (1979), 268-289. In Russian, translated in Math. USSR, Sb. 36:2 (1980), 251-271. MR 80e:53005 Zbl 0488.49029

[Mīklyukov 1980] V. M. Mīklyukov, "Capacity and a generalized maximum principle for quasilinear equations of elliptic type", Dokl. Akad. Nauk SSSR 250:6 (1980), 1318-1320. In Russian; translated in Sov. Math., Dokl. 21 (1980), 320-322. MR 83d:35019 Zbl 0553.35026

[Mitidieri and Pokhozhaev 1998] È. Mitidieri and S. I. Pokhozhaev, "Absence of global positive solutions of quasilinear elliptic inequalities", Dokl. Akad. Nauk 359:4 (1998), 456-460. In Russian; translated in Dokl. Math. 57:2 (1998), 250-253. MR 2000a:35263 Zbl 0976.35100

[Mitidieri and Pokhozhaev 1999] È. Mitidieri and S. I. Pokhozhaev, "Absence of positive solutions for quasilinear elliptic problems in $\mathbf{R}^{N}$, Tr. Mat. Inst. Steklova 227 (1999), 192-222. In Russian; translated in Proc. Steklov Inst. Math. 227:4 (1999), 186-216. MR 2001g:35082 Zbl 1056.35507

[Mitidieri and Pokhozhaev 2001] È. Mitidieri and S. I. Pokhozhaev, "A priori estimates and the absence of solutions of nonlinear partial differential equations and inequalities", Tr. Mat. Inst. Steklova 234 (2001), 1-384. In Russian; translated in Proc. Steklov Inst. Math. 234:3 (2001), 1-362. MR 2005d:35004 Zbl 0987.35002

[Ni and Serrin 1985] W.-M. Ni and J. Serrin, "Nonexistence theorems for quasilinear partial differential equations", Rend. Circ. Mat. Palermo (2) Suppl. 8 (1985), 171-185. MR 88d:35069 Zbl 0625.35028

[Ni and Serrin 1986] W.-M. Ni and J. Serrin, "Existence and nonexistence theorems for ground states of quasilinear partial differential equations: The anomalous case", Accad. Naz. Lincei 77 (1986), 231-257.

[Sun 2014] Y. Sun, "Uniqueness result for non-negative solutions of semi-linear inequalities on Riemannian manifolds", J. Math. Anal. Appl. 419:1 (2014), 643-661. MR 3217172 Zbl 1297.35297

[Sun 2015] Y. Sun, "On nonexistence of positive solutions of quasi-linear inequality on Riemannian manifolds”, Proc. Amer. Math. Soc. 143:7 (2015), 2969-2984. MR 3336621 Zbl 06428975

Received July 18, 2014. Revised March 11, 2015.

YUHUA SUN

DEPARTMENT OF MATHEMATiCS

UNIVERSITY OF BIELEFELD

D-33501 BIELEFELD

GERMANY

Current address:

SCHOOL OF MATHEMATICAL SCIENCES AND LPMC

NANKAI UNIVERSITY

94 WEIJIN ROAD

TIANJIN 300071

CHINA

sunyuhua@nankai.edu.cn 


\title{
PACIFIC JOURNAL OF MATHEMATICS
}

\author{
msp.org/pjm
}

Founded in 1951 by E. F. Beckenbach (1906-1982) and F. Wolf (1904-1989)

\section{EDITORS}

Don Blasius (Managing Editor)

Department of Mathematics

University of California

Los Angeles, CA 90095-1555

blasius@math.ucla.edu

\author{
Paul Balmer \\ Department of Mathematics \\ University of California \\ Los Angeles, CA 90095-1555 \\ balmer@math.ucla.edu \\ Robert Finn \\ Department of Mathematics \\ Stanford University \\ Stanford, CA 94305-2125 \\ finn@math.stanford.edu \\ Sorin Popa \\ Department of Mathematics \\ University of California \\ Los Angeles, CA 90095-1555 \\ popa@math.ucla.edu
}

\author{
Vyjayanthi Chari \\ Department of Mathematics \\ University of California \\ Riverside, CA 92521-0135 \\ chari@math.ucr.edu \\ Kefeng Liu \\ Department of Mathematics \\ University of California \\ Los Angeles, CA 90095-1555 \\ liu@math.ucla.edu \\ Jie Qing \\ Department of Mathematics \\ University of California \\ Santa Cruz, CA 95064 \\ qing@ cats.ucsc.edu
}

\section{PRODUCTION}

Silvio Levy, Scientific Editor, production@msp.org

\section{SUPPORTING INSTITUTIONS}

ACADEMIA SINICA, TAIPEI

CALIFORNIA INST. OF TECHNOLOGY

INST. DE MATEMÁTICA PURA E APLICADA

KEIO UNIVERSITY

MATH. SCIENCES RESEARCH INSTITUTE

NEW MEXICO STATE UNIV.

OREGON STATE UNIV.

\author{
STANFORD UNIVERSITY \\ UNIV. OF BRITISH COLUMBIA \\ UNIV. OF CALIFORNIA, BERKELEY \\ UNIV. OF CALIFORNIA, DAVIS \\ UNIV. OF CALIFORNIA, LOS ANGELES \\ UNIV. OF CALIFORNIA, RIVERSIDE \\ UNIV. OF CALIFORNIA, SAN DIEGO \\ UNIV. OF CALIF., SANTA BARBARA
}

\author{
Daryl Cooper \\ Department of Mathematics \\ University of California \\ Santa Barbara, CA 93106-3080 \\ cooper@math.ucsb.edu \\ Jiang-Hua Lu \\ Department of Mathematics \\ The University of Hong Kong \\ Pokfulam Rd., Hong Kong \\ jhlu@maths.hku.hk \\ Paul Yang \\ Department of Mathematics \\ Princeton University \\ Princeton NJ 08544-1000 \\ yang@math.princeton.edu
}

These supporting institutions contribute to the cost of publication of this Journal, but they are not owners or publishers and have no responsibility for its contents or policies.

See inside back cover or msp.org/pjm for submission instructions.

The subscription price for 2016 is US $\$ 440 /$ year for the electronic version, and $\$ 600 /$ year for print and electronic.

Subscriptions, requests for back issues and changes of subscribers address should be sent to Pacific Journal of Mathematics, P.O. Box 4163, Berkeley, CA 94704-0163, U.S.A. The Pacific Journal of Mathematics is indexed by Mathematical Reviews, Zentralblatt MATH, PASCAL CNRS Index, Referativnyi Zhurnal, Current Mathematical Publications and Web of Knowledge (Science Citation Index).

The Pacific Journal of Mathematics (ISSN 0030-8730) at the University of California, c/o Department of Mathematics, 798 Evans Hall \#3840, Berkeley, CA 94720-3840, is published twelve times a year. Periodical rate postage paid at Berkeley, CA 94704, and additional mailing offices. POSTMASTER: send address changes to Pacific Journal of Mathematics, P.O. Box 4163, Berkeley, CA 94704-0163.

PJM peer review and production are managed by EditFLOW ${ }^{\circledR}$ from Mathematical Sciences Publishers.

\section{PUBLISHED BY}

\section{mathematical sciences publishers \\ nonprofit scientific publishing}

http://msp.org/

(C) 2016 Mathematical Sciences Publishers 


\section{PACIFIC JOURNAL OF MATHEMATICS}

Volume $280 \quad$ No. $1 \quad$ January 2016

Stable capillary hypersurfaces in a wedge

JAIGYOUNG CHOE and MIYUKI KoISO

The Chern-Simons invariants for the double of a compression body

DAVID L. DUNCAN

Compactness and the Palais-Smale property for critical Kirchhoff equations in

closed manifolds

EMMANUEL HeBEy

On the equivalence of the definitions of volume of representations

SUNGWOON KIM

Strongly positive representations of even GSpin groups

YEANSU KIM

An Orlik-Raymond type classification of simply connected 6-dimensional torus manifolds with vanishing odd-degree cohomology

\section{SHINTARÔ KUROKI}

Solutions with large number of peaks for the supercritical Hénon equation

Zhongyuan LiU and ShuangJie Peng

Effective divisors on the projective line having small diagonals and small heights and 141 their application to adelic dynamics

YÛSUKE OKUYAMA

Computing higher Frobenius-Schur indicators in fusion categories constructed from inclusions of finite groups

\section{Peter Schauenburg}

Chordal generators and the hydrodynamic normalization for the unit ball

SEBASTIAN SCHLEISSINGER

On a question of A. Balog

ILYA D. SHKREDOV

Uniqueness result on nonnegative solutions of a large class of differential inequalities 241 on Riemannian manifolds

YUHUA SUN

Correction to "Closed orbits of a charge in a weakly exact magnetic field"

WILL J. MERRY 